\author{
Jorge Andreu Arasa* \\ Carlos J. Tórtola Sebastián* \\ Victoria Vera González ${ }^{* *}$
}

\title{
EL COMERCIO ELECTRÓNICO Y EL PAPEL DE LAS AGENCIAS DE PROMOCIÓN DE LA INTERNACIONALIZACIÓN. EL CASO DE ICEX. EL MERCADO CHINO
}

El comercio electrónico es lugar común de conversaciones de muchos estos días. Pero ¿es sencillo participar de los beneficios de este canal para las empresas españolas? ¿Qué papel deben jugar las Agencias de Promoción de la Internacionalización en la aproximación de la empresa a este nuevo modelo de distribución? ¿Cómo acompaña nuestra agencia, ICEX, en todo este proceso? ¿Cómo lo hace en el caso de China?

\section{E-commerce and the role of Export Promotion Agencies on it. The example of ICEX. The Chinese market \\ Currently, e-commerce discussions are widespread. Is it easy for the Spanish firms to benefit from e-commerce? What is the role that should be played by Export Promotion Agencies to support firms given this new retail model? How does ICEX help firms to manage e-commerce processes? How does ICEX do it for deals in China?}

Palabras clave: exportación, socios comerciales, promoción de exportaciones, China. Keywords: export, trading partners, export promotion, China.

JEL: F13, L81.

* Técnico Comercial y Economista del Estado.

** Directora Adjunta Comercio Electrónico ICEX.

Las opiniones expresadas solo comprometen a sus autores.

Versión de enero de 2020.

DOI: https://doi.org/10.32796/ice.2020.913.6992 


\section{Datos del mercado online}

Todos parecemos estar de acuerdo en la trascendencia del comercio electrónico como canal alternativo para abordar el proceso de venta. Las cifras parecen avalar esta apreciación: si en 2016 las ventas online representaban el $8,7 \%$ de las ventas al retail, esta cifra se espera que alcance el $14,6 \%$ en $2020^{1}$, si en 2019 el $53 \%$ de quienes navegaban por internet realizaron compras online, prácticamente un $27 \%$ de la población mundial, para 2023 se espera que este porcentaje alcance el $57 \%{ }^{2}$.

Este crecimiento se produce de forma homogénea en muchos sectores, pudiéndose observar tasas de incremento de doble dígito en los últimos años en múltiples verticales, desde la moda hasta la alimentación ${ }^{3}$.

El proceso de internacionalización de la empresa a través del comercio se ha visto rápidamente afectado también por el nuevo canal. Se observa así un importante incremento en el llamado Cross-Border E-Commerce (CBEC), venta de productos a consumidores localizados fuera del país a través del canal online, bien por medio de portales, de tiendas online de distribuidores y de plataformas (Ellia, Giuffrida y Piscitello, 2019). Así por ejemplo, el CBEC en Europa ascendió a 52.250 millones de euros en 2018, con una presencia creciente de los marketplaces en el canal (si en 2008 solo participaban en el $17 \%$ de las transacciones ahora lo hacen en un $50 \%)^{4}$.

El caso europeo podría parecer un caso particular, quizás no extrapolable, pues hay elementos determinantes de la evolución del mismo especialmente condicionados por la actuación del regulador. La Directiva sobre comercio electrónico, al armonizar reglas para potenciar el mercado interior, minoró los costes del uso del canal (Copenhagen Economics, 2007). Esta Directiva garantizó la seguridad de proveedores y clientes a

\footnotetext{
Australian Trade and Investment Comission.

2 Datos de eMarketer.

3 Datos de Statista.

4 Datos de Internet Retailer.
}

través de reglas armonizadas en relación a los requisitos de trasparencia e información para los proveedores de servicios online, comunicaciones comerciales, contratos electrónicos, limitó la responsabilidad por parte de los intermediarios y volvió al país de origen como regla para regular numerosas áreas normativas. Así, la limitación de la responsabilidad ha reducido riesgos y costes de las empresas, la armonización en la provisión de contratos ha disminuido los costes de agencia equiparando la eficiencia de un contrato offline y un contrato online, y la aplicación del principio de país de origen ha minorado la heterogeneidad entre países miembros reduciendo los costes de búsqueda.

Con todo y sin la necesidad de un marco normativo tan favorable, algo parecido ha ocurrido a nivel global, donde el CBEC representa ya un $30 \%$ del comercio electrónico (Ellia, Giuffrida y Piscitello, 2019).

\section{Oportunidad del comercio electrónico para las empresas. El caso de las plataformas}

Todo parece indicar que la nueva tecnología es una oportunidad digna de atender por las empresas, grandes y pequeñas. En el caso de las pymes, parte de la literatura económica (Ellia, Giuffrida y Piscitello, 2019) hace referencia a una mezcla de oportunidades y riesgos en el uso del canal, el comercio electrónico, como forma de internacionalización, pues a los beneficios de la reducción de costes de entrada y la disponibilidad de nuevos y más rápidos canales de comunicación se unen barreras legales, aduaneras, fiscales, problemas de marca, ... Hay incluso quien considera que el comercio online añade riesgo al proceso de internacionalización (es un hecho que vender en el canal implica algo más que crear una tienda online).

Estas barreras son diferentes en función de los países de llegada: en el caso chino las mayores dificultades a las que se enfrentan las pymes son: la gestión de los distintos canales online, la atención a los requerimientos legales, la gestión de la logística y una eficiente comunicación. Estas dificultades cambian 
cuando la pyme trata de exportar usando este canal al mercado americano, donde se enfrenta a una enorme competencia con compañías locales y otros jugadores internacionales, siendo el segundo motivo de preocupación de este tipo de empresa el uso de los canales online y la complejidad legal.

Un buen ejemplo para entender las ventajas del online es el caso de los marketplaces (Amazon, Alibaba, JD, ...), que como se ha dicho representan ya el $50 \%$ de las ventas online al retail (Internet Retailer, 2019). El uso de las plataformas reduce los costes de búsqueda de información, transacción, facilita la visibilidad y el posicionamiento internacional. Además, y a diferencia de la tienda online, no exige inversión inicial.

Alcanzar resultados positivos distribuyendo el producto a través de plataformas exige gestionar adecuadamente el catálogo de productos, agrupar o segmentar la demanda para encontrar nichos de mercado, realizar ofertas, gestionar peticiones de compra, ... Optimizar la presencia en plataformas puede exigir la creación de una tienda de marca, la coordinación de una tienda propia con la actividad en la plataforma, revisar la situación en términos de logística, analizar lo que hace la competencia, llevar a cabo un plan de marketing digital, activar una política postventa (un servicio de atención al cliente) así como realizar analítica y reporting. Sin una correcta valoración de los extremos anteriores se hará difícil que la inversión en el canal, pequeña o grande, resulte rentable. Como se puede observar, la problemática se asimila a la propia de la internacionalización con éxito en los canales tradicionales. El acceso a los mercados electrónicos supone dedicación de recursos, por otra parte siempre escasos y empleados generalmente en usos alternativos (el propio canal offline, la tienda propia online, ...). Se precisa, por tanto, cierto acompañamiento en el uso del canal (Andreu, 2019).

\section{3. ¿Por qué el sector público?}

Las dificultades a las que se enfrentan las compañías tienen que ver, al fin y al cabo, con tener una insuficiente dotación de formación, información, consultoría y promoción para hacer frente a la internacionalización empleando el comercio electrónico. La internacionalización/diversificación de canales minora el riesgo favoreciendo la creación/estabilización del empleo. Se justifica así la participación del sector público corrigiendo el equilibrio al que llega el mercado en la provisión de la mencionada formación, información, ...

\section{Diversificación de canales/mercados y minoración de riesgos. Externalidad positiva}

El modelo de selección de carteras de Markowitz, siguiendo la regla de decisión media varianza, basado en la preferencia del inversor por carteras con mayor rendimiento y menor riesgo, revisado por Sharpe para simplificar su ejecutoria estableciendo que la dependencia estadística entre los rendimientos de los diferentes activos no es directa sino indirecta, a través de terceras variables (PNB, ...) lleva a la conclusión de que la diversificación trae consigo una minoración de riesgos. El riesgo de una cartera es el resultado de agregar el riesgo sistemático o de mercado más su riesgo propio o específico. Como demuestra Sharpe, la parte de riesgo debida al mercado se puede reducir con una diversificación oportuna, pero no eliminarse. Sin embargo, el riesgo propio o específico sí podrá eliminarse, bastando para ello con escoger un número de títulos suficientemente elevado. La relación decremental entre el riesgo y el número de títulos dependerá de la eficiencia en la diversificación (Suárez Suárez, 1993).

Extrapolando al caso que nos ocupa, y salvando las distancias pues los supuestos de partida de los modelos mencionados tienen cierta especificidad, las conclusiones de los mismos podrían aplicarse al caso de otros procesos de toma de decisión en la empresa, como puedan ser la salida a un tercer mercado o el uso de un determinado canal para acceder a este. La relación decremental del riesgo condicionado por el número de países/canales dependerá de la eficiencia en la diversificación, diversificación que las pymes 
pueden no ser capaces de llevar a cabo dado su limitado acceso a financiación.

Como decíamos, la reducción del riesgo consecuencia de la diversificación facilitará una mayor contratación y estabilidad en el empleo.

\section{Acceso limitado a financiación. Información asimétrica}

La elección en procesos con información incompleta/asimétrica permite explicar por qué el mercado proveería insuficientemente con servicios de formación, información, ..., a las pymes. En el marco de una relación de Agencia (un agente actúa por cuenta de otro), uno de los agentes tiene más información que el otro. El comportamiento asimétrico de quien tiene la información explica el acceso asimétrico a la misma.

El mercado que se tomó como referencia para explicar el equilibrio subóptimo al que se llega en estos casos fue el de los coches usados (Akerlof, 1970). Al disponer el vendedor de un coche usado más información que el comprador, este último realiza una estimación del precio del mismo considerando la probabilidad de que tenga un menor valor que el que pretende el vendedor. Pero al ofrecer precios más bajos y seguir la información siendo asimétrica lo que ocurre es que los propietarios de coches buenos los dejan de ofertar. La iteración lleva a la desaparición de coches buenos en el mercado de coches usados.

Una extrapolación del modelo permite entender lo que ocurre en los mercados financieros con las pymes. Prestamista y prestatario tienen un nivel de acceso diferente a la información sobre la salud financiera de este último. El prestamista dispone de menor información y asigna una probabilidad determinada a la posibilidad de que el prestatario no pueda hacer frente al pago a vencimiento, lo que se traduce finalmente en una progresiva elevación del tipo de interés. Al igual que en el caso del mercado de coches usados, el alza de tipos de interés elimina los proyectos de inversión de los prestatarios buenos permaneciendo en el mercado solo aquellos cuyo riesgo permitiría hipotéticamente pagar el préstamo. Iterando desaparecerían los prestamistas buenos.

Para solucionar el problema los prestamistas suelen recurrir a modelos de señalización, o sencillamente al racionamiento del crédito frente a la subida natural de los tipos de interés. Esto permite que en igualdad de condiciones determinados prestatarios tengan o no acceso a financiación. El sistema de colaterales o la reducción del tamaño del crédito solo refuerzan, por lo tanto, el efecto negativo en la financiación consecuencia de la existencia de selección adversa en un marco de información asimétrica. Pymes, start-ups y autónomos pueden ser los más afectados, al no ser capaces de aportar colateral y tener probablemente un historial crediticio insuficiente.

Cobra sentido, en definitiva, la provisión de servicios de formación, información, ..., que el mercado bien no provee para determinadas empresas o de los que determinadas empresas no pueden disfrutar debido al limitado acceso a financiación.

Pero ¿qué hacen las Agencias de Promoción de la Internacionalización?

\section{Las Agencias de Promoción de la Internacionalización}

\section{El modelo general: información básica $y$ asesoramiento}

Dada la importancia creciente del canal online para vender internacionalmente, los distintos Organismos de Promoción del Comercio Exterior (OPC) han ido incorporando líneas de acción específicas relacionadas con el comercio electrónico en sus planes estratégicos. Sin embargo, varía el peso que cada agencia otorga a este aspecto y el tipo de actividades elegidas para impulsar su uso entre las empresas (detalle en Anexo I).

Muchas agencias disponen en sus webs de secciones dedicadas específicamente a E-commerce, donde agrupan su oferta de información y servicios 
(Austria - WKO5 E-Commerce und Webshop; Reino Unido - DIT6 Selling Online Overseas; Italia - ITA ${ }^{7}$ E-commerce; Alemania - GTAl ${ }^{8}$ E-Commerce und Onlinehandel; Estados Unidos - US Department of Commerce Exporting and eCommerce). En el caso de España, ICEX dispone de una web en la que agrupa sus servicios relacionados con internacionalización online bajo el programa eMarket Services (www.emarketservices.es).

Todas las agencias proporcionan información general y de mercado (datos básicos, guías, indicadores, normativa, legislación, principales plataformas, etc.) como parte de los servicios generales de información que facilitan. Algunos ejemplos de guías especializadas en comercio electrónico son los siguientes: Online Exporting: A Guide for Australian Business, de Austrade; Leitfaden E-Marketing und E-Commerce, de WKO; Exporting and Ecommerce, del US Department of Commerce.

Algunas agencias producen informes país focalizados en comercio electrónico (ej., Norway-Ecommerce, US Department of Commerce; E-commerce in China, Austrade; Le marché du e-commerce a l'Italie, Business France; E-commerce for UK small businesses selling online to the US, DIT; Ländervergleich Trends beim Konsum und im Einzelhandel, GTAI). Pero muy pocas disponen de producción propia de estudios de mercado sector/país focalizados en e-commerce (ej., Guida Digital Export Fashion \& Beauty, ITA) u otro tipo de instrumentos de información más especializados, como directorios de marketplaces (ej., Selling Online Overseas Tool, DIT).

La información se hace llegar a las empresas, además de a través de informes y referencias en sus páginas web, a través de jornadas de formación específicas, tanto presenciales como online. Encontramos oferta formativa en comercio electrónico por parte de todas las agencias, en mayor o menor medida. Algunos ejemplos de sesiones presenciales son las

5 Wirtschaftskammer Österreich, Cámara de Comercio de Austria.

6 DIT, Department for International Trade, Reino Unido.

7 ITA: Italian Trade Agency.

8 GTAI, Germany Trade and Invest. siguientes: France, Business Meetings E-commerce Chine; WKO, CHINA - Academia-Módulo 3: Marketing y ventas en China. Además de las jornadas presenciales, el formato de webinario está ampliamente extendido como instrumento de formación. Estos son algunos ejemplos: Developing a Direct-to-Customer E-Commerce Approach to Reach Customers in New Markets (DIT), Webinar: Erfolgreich im chinesischen Crossborder E-Commerce (Austrian Chamber of Commerce), así como el uso de contenidos multimedia en forma de píldoras con información básica (ej., Italian Trade Agency: E-pills e-commerce: corso online sull'export digitale).

Una importante pieza informativa son los casos de empresas, recogidos a menudo en formato video (ej., Red Herring Games exports thrills with help from Amazon, DIT).

La mayoría también disponen de servicios de asesoramiento básico, incluidos en sus servicios generales para la internacionalización, y algunas ofrecen servicios de consultoría más especializada.

En lo que respecta a actividades de promoción comercial propiamente dicha, encontramos pocos ejemplos de acciones especialmente diseñadas para este nuevo canal. Destacan en este sentido las actuaciones de Italian Trade Agency (ITA) y Department for International Trade (DIT), que trabajan en líneas más similares a las de ICEX y diferentes entre sí, como se verá a continuación.

\section{Servicios agrupados bajo un programa paraguas: el programa e-Exporting de Reino Unido}

El Department for International Trade (DIT) británico es la única agencia de las analizadas que, junto con ICEX y su programa eMarket Services, que veremos después, ha optado por agrupar los servicios relacionados con comercio electrónico bajo el paraguas de un programa que los identifica y destaca especialmente.

Se trata del programa e-Exporting (https://www. gov.uk/guidance/e-exporting), que está focalizado en 
proporcionar información y asesoramiento personalizado a las empresas británicas para vender internacionalmente a través de marketplaces.

Cuenta para ello con un directorio de 39 plataformas seleccionadas, que se encuentran entre las más importantes que operan en distintas áreas geográficas, de las que proporciona información detallada sobre requisitos de acceso y operativa. Para propiciar la participación de las empresas británicas ha firmado acuerdos con 15 de ellas que ofrecen condiciones especiales a las empresas que se registren a través del DIT (reducción de 1 o 2 puntos porcentuales de cuota sobre ventas o períodos gratuitos de participación de entre 3 y 12 meses). Dichos marketplaces son ttHigo, The Market, Spartoo, NewEgg, Linio, Fruugo, Fnac, ePrice, Darty, Catch, Trademe, Real.de, Privalia, Onbuy.com y La Redoute. Son en general plataformas orientadas a comercio B2C (Bussines to Consumer) salvo alguna excepción que contempla el canal de venta entre empresas (New Egg Business, Amazon Business, 1688.com).

El programa incluye también servicios de asesoramiento a medida para acompañar a las empresas interesadas y una formación continua a través de webinarios muy centrados en operativa en marketplaces relevantes por áreas geográficas.

\section{Servicios enfocados a promoción comercial: las acciones de ITA en Amazon (Made in Italy) y Tmall (HelloITA)}

La línea de trabajo de la Italian Trade Agency (ITA) en servicios relacionados con comercio electrónico, además de los aspectos informativos y formativos, se ha centrado en desarrollar acciones de promoción comercial colectivas específicas en determinadas plataformas, con el fin de desarrollar e impulsar la marca «Made in Italy» en el canal digital y favorecer la utilización de marketplaces por parte de las pymes italianas.

De especial relevancia son sus acciones en Amazon y TMall. Se trata en ambos casos de las plataformas
B2C con mayor volumen de ventas en los mercados en que operan y a nivel global.

Con Amazon comparten la promoción del canal "Made in Italy"9, que funciona como una brand store en los marketplaces de Amazon en Europa y Estados Unidos, apoyando así el posicionamiento de las empresas y productos italianos de los sectores de alimentación y bebidas, hogar y muebles, moda, artículos de regalo y artesanía en estas plataformas.

Con el Grupo Alibaba han desarrollado el proyecto de marketing online HellolTA, un brand hub en las plataformas de TMall y TMall Global, que funciona como escaparate de la cultura y los productos italianos para el mercado chino. A través de HellolTA se promocionan conjuntamente en estos mercados electrónicos los productos de las cerca de 100 marcas italianas que participan en este proyecto, mediante campañas específicas de KOL (Key Opinion Leaders), eventos O2O (Onlineto-Offline), marketing de contenidos y redes sociales.

Mantienen también acuerdos con las plataformas Yoox, para el sector de moda, y Ocado.com (Reino Unido) y COFCO.com (China) para los sectores de alimentos y vinos.

\section{Programas del ITC para países menos desarrollados}

Merecen una mención especial las iniciativas del International Trade Center (ITC-UNCTAD) encaminadas a impulsar el desarrollo de los instrumentos de las agencias de promoción comercial de los países menos desarrollados relacionados con transformación digital/comercio electrónico de las pymes de estos países.

En este marco, cuentan con un programa específico para impulsar el uso del comercio electrónico, denominado Ecommerce Connect, que actúa a dos niveles, con los Gobiernos y con las empresas.

\footnotetext{
9 Más información sobre las iniciativa Made in Italy https://www.ice.it/it/ amazon y HellolTA.
} 
A nivel institucional, su objetivo es ayudar a los Gobiernos a definir una estrategia y proporcionar a las instituciones implicadas el conocimiento, las capacidades y los instrumentos necesarios para promover y apoyar el desarrollo del comercio electrónico en sus países, de manera que sean capaces más tarde, con la ayuda del ITC, de formar, asesorar y proporcionar a las empresas los recursos necesarios para implementar sus proyectos de e-commerce, aportándoles los socios y soluciones que las ayuden en el proceso.

Algunos de los proyectos ya finalizados o en marcha son la plataforma www.connectuemoa.com, un marketplace para los emprendedores de los países africanos que integran la West African Economic and Monetary Union (UEMOA, por sus siglas en francés); el proyecto «Developing SMEs exports through Virtual Market Places», cuyo objetivo es que pymes de Oriente Medio y Norte de África (MENA, por sus siglas en inglés) conozcan y utilicen los marketplaces; o el proyecto «Mujeres Centroamericanas y Comercio Electrónico» con el fin de desarrollar la venta online, especialmente a través de marketplaces, de productos artesanos elaborados por mujeres de Costa Rica, El Salvador, Guatemala, Honduras, Nicaragua y Panamá.

\section{ICEX. El programa eMarket Services}

eMarket Services es el programa de ICEX orientado a ayudar a las empresas españolas a conocer y utilizar el canal online para vender en el exterior, especialmente a través de mercados electrónicos.

El servicio nació en el año 2000, el año del boom de las puntocom, como proyecto de colaboración entre varias agencias de promoción de comercio exterior. Pero en 2010 ese acuerdo se deshizo y desde entonces es un servicio exclusivo de ICEX para las pymes españolas.

eMarket Services se lanzó justo cuando aparecieron y comenzaron a desarrollarse los grandes marketplaces, Amazon, eBay o Alibaba. Enseguida ICEX entendió lo útiles que podrían ser estas plataformas para las pymes exportadoras, por las muchas ventajas que les ofrecen de cara a encontrar nuevos clientes y vender sus productos en cualquier país del mundo, con poca inversión o al menos menor inversión que la que supone visitar o implantarse en un mercado, y también con menor riesgo.

Al inicio, el objetivo principal era que las empresas fueran conscientes de la existencia y oportunidades de los marketplaces como canal adicional para la internacionalización, y facilitarles su uso a través de recursos informativos.

Ahora la situación es otra. Las empresas ya son conscientes de que deben integrar el comercio electrónico en sus estrategias de venta, dado el crecimiento espectacular que ha tenido en los últimos años a nivel mundial, y cuentan ya con cierto conocimiento de cómo funcionan, al menos de los principales marketplaces.

Así que el programa ha ido desarrollándose y cobrando envergadura a lo largo de los años siendo en estos momentos su objetivo acompañar a las empresas españolas en todo el proceso de transformación digital que necesitan para desarrollar su estrategia de comercialización online en mercados exteriores, valiéndose tanto de mercados electrónicos como de cualquier otro tipo de plataformas, ya sean tiendas online de distribuidores o retailers, como Zalando, Fallabella o Tesco, o incluso redes sociales.

En este sentido ICEX pone a disposición de las empresas un portfolio de servicios de información, asesoramiento y apoyo que cubre todas sus necesidades para definir y desplegar operativamente su estrategia de internacionalización online.

Dichos servicios se agrupan en tres bloques que se identifican con tres fases o etapas que llevarían a la empresa de ser una pyme "analógica», a una pyme «digital» conectada y operativa en los mercados internacionales online.

\section{Paso 1: Transforma tu empresa: Evaluación y formación}

El primer paso que debe dar una empresa que quiere abordar los mercados internacionales online 
es analizar su situación, sus capacidades y sus carencias. Con este fin ICEX ofrece un bloque de servicios que comienzan con una herramienta de autodiagnóstico a través de la web de eMarket Services (www.emarketservices.es). Se trata de un formulario online que, una vez cumplimentado, indica a la empresa su grado de desarrollo en las principales variables relacionadas con comercio electrónico (estrategia, tecnología, recursos humanos, logística, etc.) y la ayuda a identificar sus debilidades y necesidades de mejora.

Y para ayudarla a superar sus carencias existe un amplio catálogo de servicios de formación, como:

- Seminarios y cursos, presenciales y online, que van desde jornadas de un día sobre temas generales de comercio electrónico a talleres sobre cómo operar en un marketplace específico, como Amazon o Aliexpress (ej., Jornada AliExpress Businesses: Expande tu negocio de la mano de expertos), incluyendo formación académica de mayor alcance ofrecida por la escuela de negocios ICEX-CECO (Master en International eBusiness, Curso de Comercio Electrónico).

- Webinarios (ej., Comercio electrónico en el sector habitat en Alemania; Cómo crear una tienda online transfronteriza en WeChat).

- Tutoriales (ej., Google Market Finder, Acelera con eBay, Guía práctica de ecommerce, Cómo puedes vender más con las descripciones de producto).

- Y eventos diversos que facilitan la formación y el networking.

Aeste primer bloque de servicios, relacionado con evaluación y formación, se dedica la sección «Transforma tu empresa» en la web de eMarket Services.

\section{Paso 2: Descubre el mercado}

El segundo paso estaría orientado a conocer el mercado al que dirigirse y definir un plan de internacionalización online. Y para ello se ofrece a las empresas un bloque de servicios relacionados con «insight y estrategia», entre ellos:
- Un observatorio de tendencias e-commerce con los principales indicadores de comercio electrónico de cada país, representados sobre un mapa del mundo navegable (número de usuarios de internet y peso de las compras online respecto al PIB, principales medios de pago, grado de desarrollo del comercio electrónico, etc.).

- Además, una amplia colección de información de mercado, centrada en desarrollo digital y comercio electrónico, producida por la red de Oficinas Económicas y Comerciales en el exterior, entre ellos, más de 150 informes e-país, que analizan la situación del comercio electrónico en un país determinado e informes de mercado específicos por sector (ej., Informe e-País Japón, El comercio electrónico de vino en Alemania, El mercado de la moda online en Estados Unidos), además de 600 direcciones de interés.

- Estos recursos están accesibles gratuitamente en la web de eMarket Services previo registro, pero si la empresa necesita un análisis específico que se ajuste a sus especiales características y necesidades, ICEX ofrece un servicio de asesoramiento a medida.

La información de mercados se completa con una sección de actualidad donde además de noticias relacionadas con e-commerce, se publican casos de éxito, entrevistas y artículos especializados. De especial valor son los que publican periódicamente la Red de Expertos, socios colaboradores especialistas en materias diversas (legal, fiscal, marketing, logística, etc.) y mercados.

\section{Paso 3: Conecta con mercados electrónicos}

Tras esto, la empresa estaría en condiciones de empezar a operar en mercados electrónicos. Este sería el tercer paso, para el que ICEX ofrece un tercer bloque de servicios que están orientados a ayudar a la empresa a poner en marcha su estrategia, ayudándola a identificar las mejores soluciones para venta online en un país específico y a identificar las plataformas de venta más idóneas (marketplaces, tiendas online de distribuidores y retailers, otras webs) junto 
con asesoramiento y apoyo para implementar acciones de venta. En este sentido, eMarket Services pone a disposición de las empresas:

- El directorio de eMarket Services, un directorio con más de 1.000 mercados electrónicos, que recoge las plataformas de venta online más importantes por país y mercado, identificadas y analizadas por las Oficinas Económicas y Comerciales.

- Servicios de asesoramiento personalizado por mercados, para ayudar a las empresas a definir su estrategia en un país, elegir los canales y plataformas más adecuados y darles soporte para comenzar a operar en ellos.

Entre estos servicios se encuentra también la posibilidad de identificar proveedores o socios que puedan ayudarles a desarrollar aspectos específicos de su plan internacional (logística, marketing, medios de pago, atención a clientes...), para lo cual eMarket Services está construyendo un directorio de proveedores consultable también a través de la web.

- Un servicio de consultoría estratégica y operativa en marketplaces. En 2020 ICEX lanza este programa que apoyará a las empresas elegidas a definir y poner en marcha un plan de marketing y ventas en marketplaces.

- Todos estos servicios dirigidos a empresas individuales se complementan con acciones de promoción comercial en mercados electrónicos, mediante las cuales se busca posicionar y dar visibilidad a la oferta de productos españoles de manera agrupada, Ejemplo de ello la Tienda "Foods and Wines from Spain», un proyecto de colaboración entre ICEX y Amazon para facilitar y promocionar la venta de alimentos y bebidas españoles, de calidad y de origen $100 \%$ español, en los mercados europeos a través de los portales que Amazon tiene en España, Reino Unido, Alemania, Francia e Italia, mediante una tienda dedicada. Su objetivo es facilitar y animar a las empresas a aprovechar el canal online, además de contribuir a aumentar la presencia y la venta online internacional de productos agroalimentarios españoles a través de los mercados electrónicos, aprovechando el posicionamiento internacional de los alimentos y bebidas españoles a través de la marca sectorial de ICEX Foods and Wines from Spain.

- Acuerdos con otros marketplaces. Con el fin de facilitar la entrada de empresas españolas en mercados electrónicos, ICEX ha firmado acuerdos con otras plataformas, especialmente en China, que es un mercado prioritario para España y el número uno mundial en comercio electrónico. Así se han rubricado convenios de colaboración con los principales marketplaces que operan en el gigante asiático, entre ellos con el Grupo Alibaba, el Grupo JD.com, DHgate y Chunbo.

\section{El comercio electrónico en China}

\section{Evolución e impacto del comercio electrónico en el país}

En 2019 China se consolidó como líder mundial en el sector del comercio electrónico, superando a Estados Unidos, con unas ventas de 1,94 billones de dólares y un crecimiento del $27,3 \%$ respecto a 2018 , gracias a sus 802 millones de usuarios online, que realizan un consumo medio de casi 2.500 dólares anuales. Este volumen de ventas supone un $36,6 \%$ de las ventas minoristas de todo el país (en Estados Unidos ese porcentaje es de un $11 \%$ ), y la mitad del comercio electrónico mundial. Está previsto que en 2023 este mercado alcance los 4,1 billones de dólares y cope el $64 \%$ de las ventas minoristas del país (Cheung, 2019).

Este intenso desarrollo del comercio electrónico en China ha contribuido a impulsar su crecimiento económico, incrementando la productividad de las empresas en un $14 \%$, generando un aumento del consumo doméstico, dado que de cada 100 dólares de consumo online el $40 \%$ no se habría producido por otra vía, y fomentando el comercio transfronterizo al facilitar a las empresas involucradas en e-commerce exportar un $50 \%$ más (Kinda, 2019).

Además, el comercio electrónico está permitiendo un crecimiento más inclusivo en el ámbito rural chino, al ofrecer más opciones de consumo a sus habitantes $y$, 


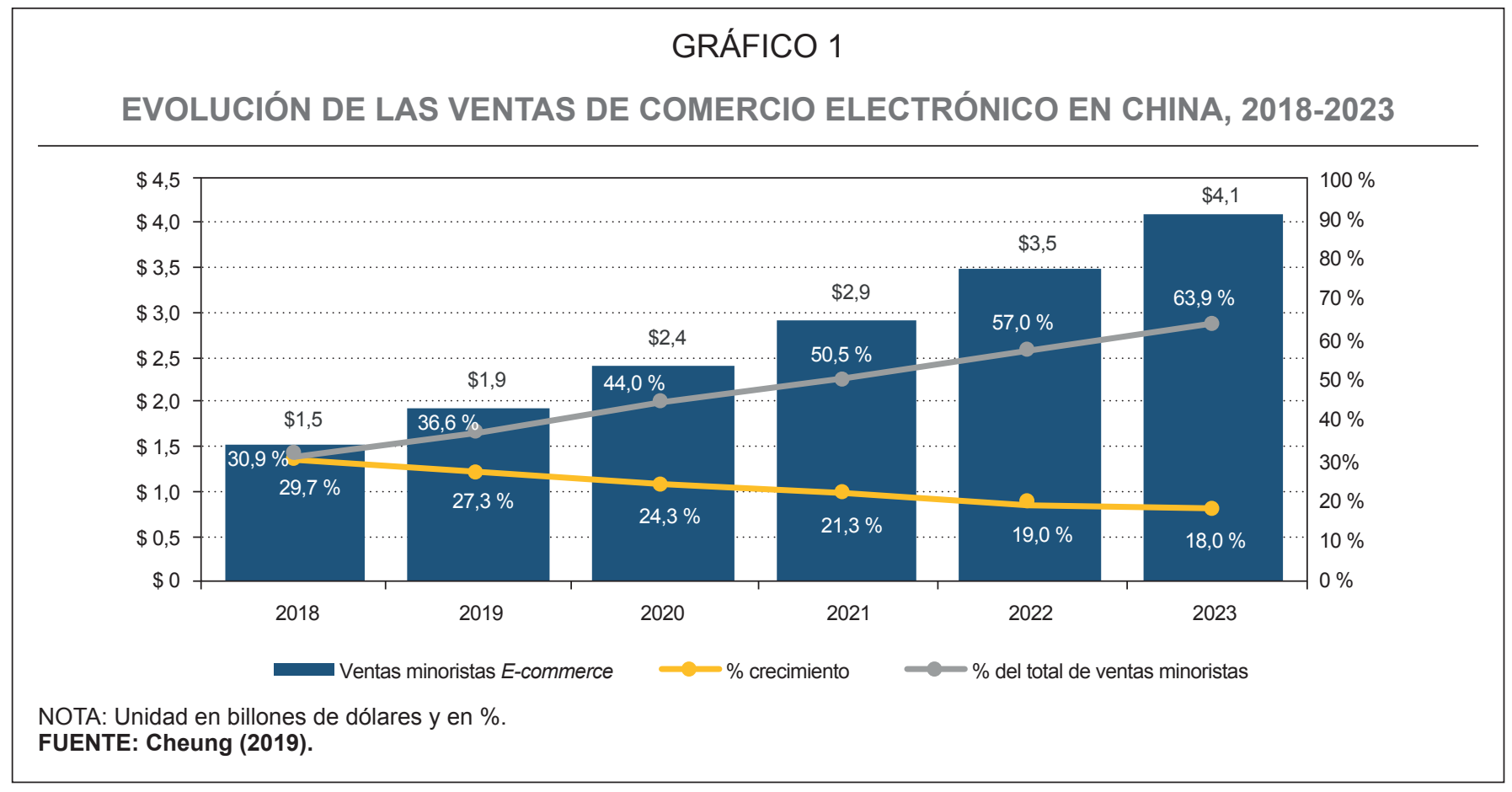

al mismo tiempo, permitir a la población rural que disponga de una fuente de ingresos estable. Uno de los casos más destacados son las «Aldeas Taobao», en las que sus habitantes venden sus productos agrícolas, artesanos o manufacturas en la plataforma Taobao del gigante de comercio electrónico Alibaba. Para ser catalogado de esta manera, el pueblo en su conjunto debe vender al menos 14 millones de euros al año o contar con más de 100 tiendas en Taobao operadas por sus habitantes. En 2019, las Aldeas Taobao ascendían a 4.310, distribuidas entre 25 provincias por todo el país (Wang, 2019).

\section{Características diferenciales del e-commerce en China}

Entre las características propias del sector del e-commerce en China destaca la penetración del móvil como instrumento para realizar las compras. En el año 2019, un $80 \%$ de las transacciones en China se realizaron a través de dispositivos móviles frente al $64,4 \%$ en el resto del mundo. En estos dispositivos móviles se integran sistemas de pago electrónico como Alipay o WeChat Pay que son aceptados ampliamente por los consumidores y las plataformas de comercio electrónico en China, agilizando el proceso de compra online que se realiza de manera rápida y segura (Cheung, 2019). Por ese motivo, el comercio electrónico en China no es otro canal de distribución más, sino que se ha integrado con las redes sociales y aplicaciones móviles para crear un entorno digital vibrante y sofisticado en el que los consumidores chinos desarrollan prácticamente la totalidad de sus actividades diarias.

Esta evolución del consumo online está obligando a las marcas a invertir en nuevas tecnologías e infraestructuras para diseñar estrategias de venta omnicanal en China en las que se combina la venta electrónica con la experiencia de compra en tiendas físicas. De hecho, pese al espectacular crecimiento del comercio electrónico en el país, en 2019 el $85 \%$ de los consumidores chinos disfrutan de la experiencia onmnicanal, frente al $80 \%$ de 2017 . Además, debido a la influencia de las 
redes sociales como WeChat, Weibo o Tik Tok, en las que los consumidores chinos permanecen conectados un $44 \%$ de su tiempo, las herramientas de marketing asociadas a estas redes sociales cobran un elevado protagonismo mediante campañas con Líderes de Opinión (KOL), o Consumidores Líderes de Opinión (KOC), o Miniprograms de WeChat ${ }^{10}$ en lo que se conoce como social commerce (Bu et al., 2019).

Otra de las características del comercio electrónico en China es el interés de los consumidores por los productos extranjeros de alta calidad. Esto se debe a los recientes escándalos en seguridad y calidad en productos locales, así como a la mayor sofisticación del consumidor chino que desea diferenciarse consumiendo productos importados. En 2018, la Encuesta de Confianza del Consumo de McKinsey indicó que el $26 \%$ de los de los consumidores chinos no estaba satisfecho con los productos domésticos por problemas de calidad y de falta de variedad (Woetzel et al., 2019).

Por este motivo, desde 2016, las autoridades chinas han aprobado diversa normativa para favorecer el comercio electrónico transfronterizo (CBEC) que permite enviar los bienes a una Zona de Libre Comercio (ZLC) en China donde quedan almacenados para su adquisición por el consumidor chino, acortándose los plazos de entrega de los productos. La normativa establecía una fiscalidad favorable, la adopción de listas positivas de productos con menores requisitos para su exportación a China, o la creación de 18 ZLC (Consulate General Netherlands, 2019).

Debido a la confusión normativa que se había generado, en 2019 entró en vigor la Ley de Comercio Electrónico con el objetivo de consolidar la normativa básica del comercio electrónico en China. Esta Ley presta atención especial a la supervisión del mercado

\footnotetext{
10 Los Miniprograms de Wechat son unas aplicaciones integradas dentro de la propia aplicación de WeChat que permite a los usuarios de WeChat realizar compras directas de productos y servicios. Además, se puede acceder a ellos directamente a través de distintas campañas de promoción que realizan los KOL o KOC en el propio WeChat. El pago también está integrado a través del propio WeChat Pay.
}

para evitar competencia desleal y a la protección de los consumidores (p. ej., problemas de propiedad intelectual), impulsando de esta manera el comercio electrónico transfronterizo (Zhang, 2018).

\section{El comercio electrónico transfronterizo en China}

El CBEC es una estrategia de venta para que las empresas extranjeras puedan obtener cuota de mercado, construir una marca y aumentar la base de consumidores. La evolución del CBEC en los últimos años ha sido positiva, alcanzando un volumen de ventas de 218.270 millones de renminbi (RMB) (aprox. 29.103 millones de euros) en 2019 con una base de consumidores de 272 millones lo que supuso un crecimiento del $35,2 \%$ y del $9,3 \%$ respectivamente. Sin embargo, pese a que puede resultar un método relativamente barato de entrada en el mercado chino, el CBEC requiere una importante inversión que puede alcanzar un millón de RMB al año (aprox. 130.000 euros). Esta inversión incluiría la adaptación de la marca al mercado chino, la logística, el marketing y posicionamiento en la plataforma, etcétera (Consulate General Netherlands, 2019).

El crecimiento del CBEC se prevé que continuará en los próximos años impulsado por la nueva clase media china que ya alcanza los 450 millones de personas. Se trata de un consumidor sofisticado y con un creciente poder adquisitivo, que muestra una preferencia por productos importados de alta calidad. Entre los productos más demandados en China a través del CBEC se encuentran: la cosmética y los productos de belleza; los productos infantiles; bebidas y alimentos; y moda, joyería y productos de lujo (Tenba Group, 2020).

\section{Actores principales}

El mercado del comercio electrónico chino está dominado por plataformas externas en las que las marcas ofrecen sus productos. Las más importantes en este momento son: Taobao, plataforma de Alibaba de C2C 
(Consumer to Consumer) $(52,5 \%$ de ratio de penetración, lanzada en 2003); Pinduoduo, especializada en ventas en grupo con descuento $(22,4 \%$ de ratio de penetración, lanzada en 2015); JD.com, especializada en B2C (22,3\% de ratio de penetración, lanzada en 2004); y Tmall, perteneciente a Alibaba y especializada en $\mathrm{B} 2 \mathrm{C}$ de productos premium $(8,4 \%$ de ratio de penetración, lanzada en 2008). Dentro del sector del B2C, JD.com y Tmall acumulan el $60 \%$ del total de transacciones online (Leung, 2019).

No obstante, cada vez más, el consumidor chino está perdiendo el miedo a operar a través de plataformas más pequeñas y menos conocidas, especializadas en un sector determinado, como serían: Chunbo (productos agroalimentarios premium), Pagoda (fruta fresca), Lalami (cosméticos), Suning.com (electrodomésticos) o Youpin (dispositivos electrónicos).

La evolución y perspectivas del mercado de comercio electrónico chino y los recientes cambios regulatorios ofrecen buenas oportunidades para las empresas españolas que deseen exportar sus productos a China. Como se ha mencionado, esto requiere un elevado esfuerzo por parte de las empresas en tiempo y recursos. ICEX trata en este marco de optimizar el acceso a este mercado minorando los costes de entrada en las plataformas chinas con acuerdos marco ${ }^{11}$ así como adaptando su instrumental (formación, información, consultoría y promoción) a las particularidades de aquel.

\section{Conclusión}

Los beneficios de la diversificación y el limitado acceso a financiación para proceder a la misma permiten entender el papel como acompañante necesario de las Agencias de Promoción de la Internacionalización como proveedores de formación, información, servicios de consultoría y promoción en el marco del comercio electrónico.

\footnotetext{
11 De momento se han firmado Convenios con JD.com, Alibaba y Chunbo.
}

En un mundo en el que las tasas de crecimiento de la distribución online son de doble dígito en gran parte de los sectores, las Agencias se enfrentan a esta labor de acompañamiento de manera muy diferente. Así ICEX ofrece una panoplia de instrumentos que permiten que el exportador español encuentre soluciones desde el mismo momento en el que se plantee utilizar el canal (autodiagnóstico), facilitando la resolución de todos los problemas con los que se pueda encontrar en el proceso.

Esta oferta de soluciones se debe adaptar a las particulares circunstancias de algunos de los mercados. Tal es el caso de China, con un elevadísimo nivel de desarrollo del canal y en el que los acuerdos con grandes plataformas deberán facilitar el acceso a los mismos de los exportadores españoles.

\section{Referencias Bibliográficas}

Akerlof, G. A. (1970). The Market for "Lemons": Quality Uncertainty and the Market Mechanism. The Quarterly Journal of Economics. The MIT Press. 84 (3) (pp. 488-500).

Andreu, J. (2019). ICEX, tu acompañamiento en la venta online internacional en marketplaces. Portal eMarket Services. Red de Expertos.

Bu, L., Wang, J., Wei Wang, K., \& Zipster, D. (2019). Consumer digital trends 2019. Discovering the next wave of growth. Shenzhen. McKinsey Digital.

Cheung, M. (2019). China Ecommerce 2019. eMarketer. New York

Consulate General of the Kingdom of the Netherlands (2019). Cross-Border E-Commerce Guidebook. Second Edition. Shanghai. Consulate General of the Kingdom of the Netherlands in Shanghai.

Copenhagen Economics (2007). Study on the Economic impact of the Electronic Commerce Directive. European Commission.

Ellia, S., Giuffrida, M., \& Piscitello, L. (2019). Does e-Commerce facilitate or complicate SMEs' internationalisation? Información Comercial Española (ICE), Revista de Economía (909), 61-73.

Internet Retailer (2019). Online Marketplaces Report, Vertical Web Media, LLC. https://www.digitalcommerce360.com/ product/online-marketplaces-report/

Kinda, T. (2019). E-commerce as a Potential New Engine for Growth in Asia. Washington DC. International Monetary Fund. Working Paper WP/19/135. 
Leung, Y. (2019). Top 5 China Shopping Apps: China Ecommerce Trends. Dragon Social. Retrieved on January 30, 2020 from https://www.dragonsocial.net/blog/top-chinashopping-apps-china-ecommerce/

Suárez Suárez, A. S. (1993). Decisiones óptimas de inversión y financiación en la empresa, 453-492. Madrid: Editorial Pirámide.

Tenba Group (2020). The 2020 China Cross Border Ecommerce Insights. Tenba Group. Retrieved on January 30, 2020 from https://tenbagroup.com/chinese-marketing/the-2020-chinacross-border-ecommerce-insights/
Wang, J. (2019). Taobao Villages driving "inclusive growth" in rural China. ALIZILA. Retrieved on January 30, 2020 from https://www.alizila.com/taobao-villages-driving-inclusive-growth-rural-china/

Woetzel, J., Seong, J., Leung, N., Ngai, J., Manyika, J., Madgavkar, A., Lund, S., \& Mironenko, A. (2019). China and the world: Inside the dynamics of a changing relationship. McKinsey Global Institute. Shanghai.

Zhang, L. (2018). China: E-Commerce Law Passed. The Library of Congress. Washington DC: Global Legal Monitor. 
ANEXO I

SERVICIOS E-COMMERCE DE LAS PRINCIPALES OPC

\begin{tabular}{|c|c|c|c|c|c|c|c|}
\hline País & Estados Unidos & Italia & Reino Unido & Australia & Francia & Alemania & España \\
\hline Información básica & Sí & Sí & Sí & Sí & No & Sí & Sí \\
\hline $\begin{array}{l}\text { Informes país } \\
\text { especializados en } \\
\text { e-commerce }\end{array}$ & $\begin{array}{l}\text { Sí. Breve reseña } \\
\text { en sus Country } \\
\text { Commercial } \\
\text { Guides }\end{array}$ & $\begin{array}{l}\text { Sí. Fichas } \\
\text { básicas de } \\
\text { Statista }\end{array}$ & $\begin{array}{l}\text { Sí. Algunos } \\
\text { breves } \\
\text { artículos }\end{array}$ & $\begin{array}{l}\text { Sí, para paí- } \\
\text { ses ASEAN }\end{array}$ & $\begin{array}{l}\text { Sí. Sobre } \\
\text { ASEAN }\end{array}$ & $\begin{array}{l}\text { Sí. Artículos } \\
\text { breves }\end{array}$ & Sí \\
\hline $\begin{array}{l}\text { Informes de mercado } \\
\text { especializados en } \\
\text { e-commerce }\end{array}$ & $\begin{array}{l}\text { No. Artículos } \\
\text { breves en la web } \\
\text { con enlaces a } \\
\text { recursos externos }\end{array}$ & $\begin{array}{l}\text { Sí, pero } \\
\text { generales, no } \\
\text { por países }\end{array}$ & No & No & Sí. Fichas & $\begin{array}{l}\text { Sí, en forma } \\
\text { de artículos } \\
\text { breves }\end{array}$ & $\begin{array}{l}\text { Sí. Estudios } \\
\text { de mercado }\end{array}$ \\
\hline $\begin{array}{l}\text { Formación- Tutoriales } \\
\text { online }\end{array}$ & Sí & Sí & Sí & $\begin{array}{l}\text { Sí. Guía } \\
\text { online }\end{array}$ & No & Sí & Sí \\
\hline $\begin{array}{l}\text { Formación- Jornadas, } \\
\text { Seminarios y Cursos }\end{array}$ & $\begin{array}{l}\text { No constan en su } \\
\text { oferta para } 2020\end{array}$ & Sí & Sí & $\begin{array}{l}\text { No constan } \\
\text { en su oferta } \\
\text { para } 2020\end{array}$ & Sí & Sí & Sí \\
\hline $\begin{array}{l}\text { Instrumentos } \\
\text { autodiagnóstico y } \\
\text { checklists }\end{array}$ & No & No & No & Sí, checklists & No & No & Sí \\
\hline Asesoramiento básico & $\begin{array}{l}\text { No, fuera de su } \\
\text { oferta general }\end{array}$ & $\begin{array}{l}\text { No, fuera } \\
\text { de su oferta } \\
\text { general }\end{array}$ & Sí & $\begin{array}{l}\text { No, fuera } \\
\text { de su oferta } \\
\text { general }\end{array}$ & $\begin{array}{l}\text { No, fuera } \\
\text { de su oferta } \\
\text { general }\end{array}$ & $\begin{array}{l}\text { No, fuera } \\
\text { de su oferta } \\
\text { general }\end{array}$ & Sí \\
\hline $\begin{array}{l}\text { Consultoría } \\
\text { especializada a medida }\end{array}$ & $\begin{array}{l}\text { No, fuera de su } \\
\text { oferta general }\end{array}$ & $\begin{array}{l}\text { No, fuera } \\
\text { de su oferta } \\
\text { general }\end{array}$ & Sí & $\begin{array}{l}\text { No, fuera } \\
\text { de su oferta } \\
\text { general }\end{array}$ & $\begin{array}{l}\text { No, fuera } \\
\text { de su oferta } \\
\text { general }\end{array}$ & $\begin{array}{l}\text { No, fuera } \\
\text { de su oferta } \\
\text { general }\end{array}$ & Sí \\
\hline Programas de ayuda & $\begin{array}{l}\text { No, fuera de su } \\
\text { oferta general }\end{array}$ & $\begin{array}{l}\text { No, fuera } \\
\text { de su oferta } \\
\text { general }\end{array}$ & $\begin{array}{l}\text { No, fuera } \\
\text { de su oferta } \\
\text { general }\end{array}$ & $\begin{array}{l}\text { No, fuera } \\
\text { de su oferta } \\
\text { general }\end{array}$ & $\begin{array}{l}\text { No, fuera } \\
\text { de su oferta } \\
\text { general }\end{array}$ & $\begin{array}{l}\text { No, fuera } \\
\text { de su oferta } \\
\text { general }\end{array}$ & Sí \\
\hline $\begin{array}{l}\text { Acciones de promoción } \\
\text { comercial }\end{array}$ & $\begin{array}{l}\text { No, fuera de su } \\
\text { oferta general }\end{array}$ & Sí & Sí & Sí & $\begin{array}{l}\text { No, fuera } \\
\text { de su oferta } \\
\text { general }\end{array}$ & $\begin{array}{l}\text { No, fuera } \\
\text { de su oferta } \\
\text { general }\end{array}$ & Sí \\
\hline $\begin{array}{l}\text { Acuerdos con } \\
\text { marketplaces y } \\
\text { otras plataformas de } \\
\text { e-commerce }\end{array}$ & $\begin{array}{l}\text { No, fuera de su } \\
\text { oferta general }\end{array}$ & Sí & Sí & Sí & $\begin{array}{l}\text { No, fuera } \\
\text { de su oferta } \\
\text { general }\end{array}$ & $\begin{array}{l}\text { No, fuera } \\
\text { de su oferta } \\
\text { general }\end{array}$ & Sí \\
\hline $\begin{array}{l}\text { Directorio de } \\
\text { marketplaces }\end{array}$ & No & No & $\begin{array}{l}\text { Sí, con } 39 \\
\text { e-markets }\end{array}$ & No & No & No & $\begin{array}{l}\text { Sí, con } \\
1.017 \\
\text { e-markets }\end{array}$ \\
\hline $\begin{array}{l}\text { Directorio de } \\
\text { proveedores }\end{array}$ & Sí & No & No & No & No & No & Sí \\
\hline Elaboración & ia. & & & & & & \\
\hline
\end{tabular}

